\title{
Background error covariance functions for vector wind analyses using Doppler-radar radial-velocity observations
}

\author{
By QIN XU ${ }^{1 *}$, SHUN LIU ${ }^{2,4}$ and MING XUE ${ }^{3,4}$ \\ ${ }^{1}$ NOAA/National Severe Storms Laboratory, Norman, Oklahoma, USA \\ ${ }^{2}$ Cooperative Institute for Mesoscale Meteorological Studies, University of Oklahoma, USA \\ ${ }^{3}$ School of Meteorology, University of Oklahoma, USA \\ ${ }^{4}$ Center for Analysis and Prediction of Storms, University of Oklahoma, USA
}

(Received 29 September 2005; revised 19 July 2006)

\section{SUMMARY}

A two-dimensional form of cross-covariance function between the radar radial- and tangential-components (with respect to the direction of radar beam) of background wind errors is derived. Like the previously derived auto-covariance function for the radial component, this cross-covariance function is homogeneous but nonisotropic in the horizontal. The auto- and cross-covariance functions are used with the statistical interpolation technique to perform a vector wind analysis from Doppler radial-velocity observations on a conical surface of low-elevation radar-scans. The structures of the two covariance functions are compared and interpreted in terms of the influence of a single-point radial-velocity observation on the analysed vector wind field. The utility and value of these covariance functions are demonstrated through analysis experiments that use either simulated radialvelocity data from idealized flows or real radar observations. The results of the statistical interpolation scheme utilizing the proposed covariance functions are shown to be superior to the results of traditional VAD technique. The proposed technique can actually be considered a generalization of the traditional VAD technique.

KEYWORDS: Cross-covariance Mesoscale Statistical interpolation

\section{INTRODUCTION}

Many weather forecast offices, especially those of the US National Weather Service, make extensive use of Doppler-radar observations for severe storm detection and nowcasting. Based on single radial component of velocity observations by radar, low-levelwind conditions and related fine features (such as convergence and rotation) are usually subjectively assessed by forecasters. Timely and accurate analyses of low-level-wind conditions at high resolutions are critical for severe-weather warning and monitoring. Analysis tools that quantitatively estimate the unobserved cross-beam wind-component are still lacking at individual forecasting offices. To meet this need, a storm-targeted radar-wind-retrieval system (STWR) is being developed (Zhang et al. 2005) based on the two-dimensional simple adjoint (2dSA) technique (Qiu and Xu 1992; Xu et al. $1994,1995,2001$ a). In this system, the 2dSA is used to retrieve high-resolution stormwinds directly on each conical surface of low-elevation radar-scans. In comparison with existing four-dimensional and three-dimensional adjoint techniques for high-resolution single-Doppler-wind retrievals (e.g., Sun and Crook 1994, 2001; Gao et al. 2001; Xu et al. 2001b), the 2dSA is more suitable for the purpose of our STWR system, because vector winds need to be retrieved directly on each two-dimensional conical surface and overlaid with the radar reflectivity (or radial-velocity) image on the corresponding conical surface for easy and direct use by forecasters (see, e.g., Fig. 6 of Zhang et al. (2005)). Typical targets are storms, mesocyclones and tornadoes; to track them effectively at high resolutions (down to $250 \mathrm{~m}$ ), and with a computational efficiency required by realtime applications, the $2 \mathrm{dSA}$ uses a small domain $\left(\sim 20 \times 20 \mathrm{~km}^{2}\right)$ in a moving frame following the tracked feature (Gal-Chen 1982; Zhang and Gal-Chen 1996; Liou 1999; Liu et al. 2004). Because the $2 \mathrm{dSA}$ has a small retrieval domain, it requires suitable

* Corresponding author: Dr Qin Xu, National Severe Storms Laboratory, 120 David L. Boren Blvd., Norman, Oklahoma 73072, USA. e-mail: Qin.Xu@noaa.gov

(c) Royal Meteorological Society, 2006. 


\title{
Background Error Covariance Functions for Vector Wind Analyses Using Doppler Radar Radial-Velocity Observations
}

\author{
Qin $\mathrm{Xu}^{1}$, Shun $\mathrm{Liu}^{2,4}$ and Ming Xue 3,4 \\ ${ }^{1}$ NOAA/National Severe Storms Laboratory, Norman, Oklahoma, USA \\ ${ }^{2}$ Cooperative Institute for Mesoscale Meteorological Studies, \\ ${ }^{3}$ School of Meteorology and ${ }^{4}$ Center for Analysis and Prediction of Storms, \\ University of Oklahoma, USA
}

(Quart. J. Roy. Meteor. Soc., First submission September 2005, Accepted August 2006)

Corresponding author address:

Dr. Qin Xu

National Severe Storms Laboratory

120 David L. Boren Blvd., Norman, Oklahoma 73072, USA

E-mail: Qin.Xu@noaa.gov

\begin{abstract}
A two-dimensional form of cross-covariance function between the radar radial- and tangential-components (with respect to the direction of radar beam) of background wind errors is derived. Like the previously derived auto-covariance function for the radial-component, this cross-covariance function is homogeneous but non-isotropic in the horizontal. The auto- and cross-covariance functions are used with the statistical interpolation technique to perform a vector wind analysis from Doppler radial-velocity observations on a conical surface of lowelevation radar scans. The structures of the two covariance functions are compared and interpreted in terms of the influence of a single-point radial-velocity observation on the analyzed vector wind field. The utility and value of these covariance functions are demonstrated through analysis experiments that use either simulated radial-velocity data from idealized flows or real radar observations. The results of the statistical interpolation scheme utilizing the proposed covariance functions are shown to be superior to the results of traditional VAD technique. The proposed technique can actually be considered a generalization of the traditional VAD technique.
\end{abstract}

KEYWORDS: Error covariance Doppler radar wind Vector wind analysis 


\section{Introduction}

Many weather forecast offices, especially those of the U.S. National Weather Service, make extensive use of Doppler radar observations for severe storm detection and nowcasting. Based on single radial component of velocity observations by radar, low-level wind conditions and related fine features (such as convergence and rotation) are usually subjectively assessed by forecasters. Timely and accurate analyses of low-level wind conditions at high resolutions are critical for severe weather warning and monitoring. Analysis tools that quantitatively estimate the unobserved cross-beam wind component are still lacking at individual forecasting offices. To meet this need, a storm-targeted radar wind retrieval (STWR) system is being developed (Zhang et al. 2005) based on the two-dimensional simple adjoint (2dSA) technique (Qiu and Xu 1992; $\mathrm{Xu}$ et al. 1994, 1995, 2001a). In this system, the 2dSA is used to retrieve high-resolution storm winds directly on each conical surface of low-elevation radar scans. In comparison with existing four-dimensional and three-dimensional adjoint techniques for high-resolution single-Doppler wind retrievals (e.g., Sun and Crook 1994, 2001; Gao et al. 2001; Xu et al 2001b), the 2dSA is more suitable for the purpose of our STWR system, because vector winds need to be retrieved directly on each two-dimensional conical surface and overlaid with the radar reflectivity (or radial-velocity) image on the corresponding conical surface for easy and direct use by the forecasters (see, e.g., Fig. 6 of Zhang et al. 2005). To effectively track the targeted storm, mesocyclone or tornado at very high resolutions (up to $250 \mathrm{~m}$ ) with a computational efficiency required by real-time applications, the $2 \mathrm{dSA}$ uses a small domain $\left(\sim 20 \times 20 \mathrm{~km}^{2}\right)$ in a moving frame following the tracked feature (Gal-Chen 1982; Zhang and Gal-Chen 1996; Liou 1999; Liu et al. 2004). Because the $2 \mathrm{dSA}$ has a small retrieval domain, it requires suitable analyses of winds at the meso- $\beta$ and meso- $\gamma$ scales, or scales larger than those handled by the small domain of $2 \mathrm{dSA}$ analysis, to provide background and boundary constraints.

The 2dSA method retrieves the unobserved cross-beam winds by tracking, using a system involving the time integration of a two-dimensional advection equation and its adjoint, the movement of high-resolution radar echoes and/or radial velocity patterns. Due to this nature, the $2 \mathrm{dSA}$ requires a high-resolution grid for it to work properly. In principle, the $2 \mathrm{dSA}$ can be applied directly to a high-resolution grid over the entire mesoscale domain, but the retrieval tends to be severely impacted by the presence of large data holes in the analysis domain and the computational cost is much higher. Further, a single large domain also prevents us from following multiple storm cells using moving coordinate frame (as we do with the 2dSA analysis). For this reason, we seek to develop an alternative technique that will provide efficient analysis at the meso- $\beta$ and meso- $\gamma$ scales, and to use it to drive the nested small-domain $2 \mathrm{dSA}$ analysis. Such mesoscale analyses can also be used directly by forecasters for broader scale applications.

Among the existing, relatively simple and efficient, techniques, various least-squares methods had been proposed for three-dimensional wind analyses from single-Doppler observations (Laroche and Zawadzki 1994; Shapiro et al. 1995; Qiu and Xu 1996; Zhang and Gal-Chen 1996; Liou and Luo 2001; Weygandt et al. 2002). These least-squares methods, while relatively simple and efficient, are, however, not particularly suitable for our purpose because the vector wind retrievals are not performed directly on the two-dimensional conical surfaces of radar scans.

The radar observations are usually much denser on the conical surfaces of radar scan than in the vertical between conical surfaces. Performing the wind analyses directly on the conical surfaces allows us to retain the observation information at the highest resolution possible. For our purpose of providing boundary information for the nested conical-surface $2 \mathrm{dSA}$ analysis, the 
use of the conical surface is a natural choice, for both accuracy and efficiency considerations. Performing the analysis directly on the conical surfaces also removes us from the need to worry about the possible inaccuracy in the height determination of the radar beams affected by the atmospheric refractivity gradients especially in the presence of a strong temperature and/or humidity inversion (Gao et al. 2006).

In this paper, we develop a method for performing the desired wind analysis on the radar conical surfaces, based on the statistical interpolation technique (Daley 1991). The statistical interpolation, also commonly referred to as the optimal interpolation, was a widely used method in operational atmospheric data assimilation (Lorenc 1981; Parrish and Derber 1992; Barker 1992). In the standard statistical interpolation, the analysis adds an increment or correction to the background (typically provided by a model prediction from the previous assimilation cycle) on the model grid. For our purpose, the analysis is performed on the conical surface. For this reason, the background error covariance functions need to be formulated in the polar coordinates on the conical surface. As will be shown in sections 3 and 4 of this paper, formulating the background error covariance functions in the polar coordinates on the conical surface will not only simplify the covariance computation and improve the analysis accuracy but also facilitate direct examination of the correlation structures represented by the error covariance functions on the conical surface.

In the previous study of $\mathrm{Xu}$ and Gong (2003, henceforth referred to as XG03), an autocovariance function was derived for the radial component of the background wind projected onto the radar beam direction at a low elevation. As shown in XG03, the derived auto-covariance function can be used with the statistical interpolation for radial-velocity analysis on the conical surface. As a sequel to XG03, this paper will derive the cross-covariance function between the radial and cross-beam tangential components of the background wind errors and then combine it with the previously derived auto-covariance function to form a complete set of covariance functions, which are used to perform vector wind analysis on the conical surface.

Our method can be considered an extension of the traditional VAD method (Lhermitte and Atlas 1961; Browning and Wexler 1968), which is a least-squares method in the onedimensional periodic space along a constant-range circle. Owing to its simplicity, the VAD is extremely efficient but assumes that the vector wind field is horizontally uniform at the level of range circle. Our extension allows the analyzed vector wind to vary along the circle therefore relaxes the uniform wind assumption in the VAD. Furthermore, our method provides a twodimensional wind analysis instead of one VAD vector wind on each range circle.

The rest of this paper is organized as follows. The cross-covariance function is first derived in section 2 and the method of analysis based on the covariance functions is described in section 3 . The structures of the auto- and cross-covariance functions are compared and interpreted in section 4 in terms of the influence of a single-point radial-velocity observation on the analyzed vector wind field. The utilities of these covariance functions are further demonstrated for the vector wind analyses with idealized as well as real radar data in section 5. Conclusions follow in section 6 .

\section{Background error covariance functions for radial and tangential velocities}

As in XG03, the random vector fields of background wind errors are assumed to be unbiased, homogeneous and isotropic in the horizontal (see section 2 of $\mathrm{Xu}$ and Wei 2001; Part I of Panchev 1971) or, approximately, on a conical surface of low-elevation radar scans (with the elevation angle $\left.<5^{\circ}\right)$. The projection of a vector field of background wind error onto the conical surface is denoted by $\mathbf{v}(x, y)=(u(x, y), v(x, y))^{\mathrm{T}}$ or, simply, $\mathbf{v}=(u, v)^{\mathrm{T}}$ where $(\bullet)^{\mathrm{T}}$ is the transpose 
of $(\bullet)$. The symbol $\mathbf{v}_{\mathrm{i}}(i=1,2)$ denotes the value of $\mathbf{v}$ at point $\mathbf{x}_{\mathrm{i}}=\left(x_{\mathrm{i}}, y_{\mathrm{i}}\right)^{\mathrm{T}}$. The covariance of $\mathbf{v}$ between any two points $(i=1,2)$ is a second-order tensor defined by $\mathbf{C}_{\mathbf{v v}}=\left\langle\mathbf{v}_{1} \mathbf{v}_{2} \mathrm{~T}_{>}\right.$, where $<(\bullet)>$ denotes the expectation (statistical mean) of $(\bullet)$. The velocity vector $\mathbf{v}_{\mathbf{i}}$ can be projected onto the $l$-direction (that is, the direction from point $\mathbf{x}_{1}$ to point $\mathbf{x}_{2}$ ) and onto the $t$-direction that is perpendicular to the $l$-direction with the positive direction pointing to the left. The resulting components are denoted by $v_{l i}$ and $v_{t i}$, respectively. These two components are related to $\mathbf{v}_{\mathbf{i}}=\left(u_{\mathrm{i}}\right.$, $\left.v_{\mathrm{i}}\right)^{\mathrm{T}}$ by $\left(v_{l i}, v_{t i}\right)^{\mathrm{T}}=\boldsymbol{R} \mathbf{v}_{\mathrm{i}}$ where $\boldsymbol{R}=\boldsymbol{R}(\alpha)$ is the rotational matrix that rotates the $x$-axis to the $l$ direction and $\alpha=\tan ^{-1}\left[\left(y_{2}-y_{1}\right) /\left(x_{2}-x_{1}\right)\right]$ is the angle of vector $\mathbf{x}_{2}-\mathbf{x}_{1}$ with respect to the $x$-axis, measured positive counterclockwise (see Fig. 1 of XG03). Note that $\boldsymbol{R}^{-1}=\boldsymbol{R}(-\alpha)=\boldsymbol{R}^{\mathrm{T}}$ and thus $\mathbf{v}_{\mathrm{i}}$ $=\boldsymbol{R}^{\mathrm{T}}\left(v_{l i}, v_{t i}\right)^{\mathrm{T}}$. Substituting this into $\mathbf{C}_{\mathbf{v v}}=\left\langle\mathbf{v}_{1} \mathbf{v}_{2}^{\mathrm{T}}>\right.$ gives $\mathbf{C}_{\mathbf{v v}}=\boldsymbol{R}^{\mathrm{T}} \mathbf{C} \boldsymbol{R}$ or, as in (2.2)-(2.4) of $\mathrm{XG} 03$,

$$
\begin{aligned}
& <u_{1} u_{2}>=C_{l l}(r) \cos ^{2} \alpha+C_{t t}(r) \sin ^{2} \alpha, \\
& <v_{1} v_{2}>=C_{l l}(r) \sin ^{2} \alpha+C_{t t}(r) \cos ^{2} \alpha, \\
& <u_{1} v_{2}>=<v_{1}, u_{2}>=\left[C_{l l}(r)-C_{t t}(r)\right] \sin \alpha \cos \alpha .
\end{aligned}
$$

Here, $C_{l l}(r)$ and $C_{t t}(r)$ are the diagonal components of $\mathbf{C}, r=\left|\mathbf{x}_{2}-\mathbf{x}_{1}\right|=\left[\left(x_{2}-x_{1}\right)^{2}+\left(y_{2}-y_{1}\right)^{2}\right]^{1 / 2}$, and the isotropy is assumed in the strict sense for both rotations and mirror reflections, so $C_{l t}(r)$ $=C_{t l}(r)=0$ for non-diagonal components of $\mathbf{C}$ (see section 2 and Fig. 3c of Xu and Wei 2001).

The radial and tangential components of $\mathbf{v}_{\mathbf{i}}$ viewed from the radar located at the origin $(x=y$ $=0$ ) can be expressed, respectively, as

$$
\begin{aligned}
& v_{\mathrm{ri}}=u_{\mathrm{i}} \cos \beta_{i}+v_{\mathrm{i}} \sin \beta_{i}, \\
& v_{\tau \mathrm{i}}=-u_{\mathrm{i}} \sin \beta_{i}+v_{\mathrm{i}} \cos \beta_{i},
\end{aligned}
$$

where $\beta_{i}=\tan ^{-1}\left(y_{\mathrm{i}} / x_{\mathrm{i}}\right)$. As shown in (2.6) of XG03, the covariance function of $v_{\mathrm{r}}$ is defined by $C_{\mathrm{rr}}\left(\mathbf{x}_{1}, \mathbf{x}_{2}\right)=\left\langle v_{\mathrm{r} 1} v_{\mathrm{r} 2}>\right.$ and can be expressed in terms of $C_{l l}(r)$ and $C_{t t}(r)$. The cross-covariance functions between $v_{\mathrm{r}}$ and $v_{\tau}$ are defined by $C_{\mathrm{r} \tau}\left(\mathbf{x}_{1}, \mathbf{x}_{2}\right)=\left\langle v_{\mathrm{r} 1} v_{\tau 2}>\right.$ and $C_{\tau \mathrm{r}}\left(\mathbf{x}_{1}, \mathbf{x}_{2}\right)=<v_{\tau 1} v_{\mathrm{r} 2}>$. For the vector wind analyses developed in this paper, these functions also need to be expressed in terms of $C_{l l}(r)$ and $C_{t t}(r)$. By using (2.1)-(2.2), one can verify that

$$
\begin{aligned}
& C_{\mathrm{r} \tau}\left(\mathbf{x}_{1}, \mathbf{x}_{2}\right)=C_{\tau \mathrm{r}}\left(\mathbf{x}_{2}, \mathbf{x}_{1}\right)=<v_{\mathrm{r} 1} v_{\tau 2}> \\
& \quad=<\left(u_{1} \cos \beta_{1}+v_{1} \sin \beta_{1}\right)\left(-u_{2} \sin \beta_{2}+v_{2} \cos \beta_{2}\right)> \\
& \quad=0.5\left[\mathrm{C}_{l l}(r)+C_{t t}(r)\right] \sin \left(\Delta \beta_{1}-\Delta \beta_{2}\right)-0.5\left[C_{l l}(r)-C_{t t}(r)\right] \sin \left(\Delta \beta_{1}+\Delta \beta_{2}\right),
\end{aligned}
$$

where $\Delta \beta_{1}=\beta_{1}-\alpha$ and $\Delta \beta_{2}=\beta_{2}-\alpha$ are the angles of vectors $\mathbf{x}_{1}$ and $\mathbf{x}_{2}$, respectively, with respect to vector $\mathbf{x}_{2}-\mathbf{x}_{1}$ (measured positive counterclockwise). It is also easy to verify the following limiting cases of (2.3):

$$
\begin{aligned}
& C_{\mathrm{r} \tau}\left(\mathbf{x}_{1}, \mathbf{x}_{2}\right)=0 \quad \text { as } \Delta \beta_{1} \text { and } \Delta \beta_{2}=0 \text { or } \pi ; \\
& C_{\mathrm{r} \tau}\left(\mathbf{x}_{1}, \mathbf{x}_{2}\right)=-C_{l l}(r) \sin \left(\Delta \beta_{2}\right) \quad \text { as } \Delta \beta_{1}=0 ; \\
& C_{\mathrm{r} \tau}\left(\mathbf{x}_{1}, \mathbf{x}_{2}\right)=C_{t t}(r) \sin \left(\Delta \beta_{1}\right) \quad \text { as } \Delta \beta_{2}=0 ;
\end{aligned}
$$




$$
\begin{aligned}
& C_{\mathrm{r} \tau}\left(\mathbf{x}_{1}, \mathbf{x}_{2}\right)=C_{t t}(r) \cos \left(\Delta \beta_{2}\right) \quad \text { as } \Delta \beta_{1}=\pi / 2 ; \\
& C_{\mathrm{r} \tau}\left(\mathbf{x}_{1}, \mathbf{x}_{2}\right)=-C_{l l}(r) \cos \left(\Delta \beta_{1}\right) \text { as } \Delta \beta_{2}=\pi / 2 \text {. }
\end{aligned}
$$

The error covariance functions estimated for the background winds at $850 \mathrm{hPa}$ in Fig. 1 of $\mathrm{Xu}$ and Wei (2001) can be approximated by the following analytical forms (see Fig. 2 of XG03):

$$
\begin{aligned}
& C_{l l}(r)+C_{t t}(r)=2 \sigma^{2} \exp \left[-r^{2} /\left(2 L^{2}\right)\right], \\
& C_{l l}(r)-C_{t t}(r)=2 a \sigma^{2} r^{2}\left(b L^{2}\right)^{-1} \exp \left[-r^{2} /\left(2 b L^{2}\right)\right],
\end{aligned}
$$

where $2 \sigma^{2}$ is the variance of $\mathbf{v}, L$ is the decorrelation length scale for the random vector field $\mathbf{v}$, and $a$ and $b$ are, respectively, the non-dimensional parameters related to the variance ratio $\left(\sigma_{\text {div }} / \sigma_{\text {rot }}\right)^{2}$ and decorrelation length ratio $L_{\text {div }} / L_{\text {rot }}$ between the divergent and rotational parts of $\mathbf{v}$ (see section 3 of XG03). Substituting (2.4) into (2.6) of XG03 and (2.3) gives

$$
\begin{gathered}
C_{\mathrm{rr}}\left(\mathbf{x}_{1}, \mathbf{x}_{2}\right)=\sigma^{2} \exp \left[-r^{2} /\left(2 L^{2}\right)\right]\left[\cos \left(\Delta \beta_{1}-\Delta \beta_{2}\right)-a r^{2}\left(b L^{2}\right)^{-1} \cos \left(\Delta \beta_{1}+\Delta \beta_{2}\right)\right] \\
C_{\mathrm{r \tau}}\left(\mathbf{x}_{1}, \mathbf{x}_{2}\right)=C_{\tau \mathrm{r}}\left(\mathbf{x}_{2}, \mathbf{x}_{1}\right)=\sigma^{2} \exp \left[-r^{2} /\left(2 L^{2}\right)\right]\left[\sin \left(\Delta \beta_{1}-\Delta \beta_{2}\right)-a r^{2}\left(b L^{2}\right)^{-1} \sin \left(\Delta \beta_{1}+\Delta \beta_{2}\right)\right] .
\end{gathered}
$$

According to (3.5)-(3.7) of XG03, $a=0$ and $b=1$ correspond to $\sigma_{\mathrm{div}} / \sigma_{\mathrm{rot}}=1$ and $L_{\mathrm{div}} / L_{\mathrm{rot}}=1$. These parameter values will be used for the analyses in this paper. This choice of parameter values assumes that the divergent and rotational parts of the background wind have about the same error variance and decorrelation length scale, so it should be acceptable for wind analyses at the mesoscale and even smaller scales.

\section{Method of analysis}

The analysis is performed on the conical surface of the radar scans and the analysis domain (on the conical surface) is discretized in the same way as a regular horizontal grid used by the background field. On this discretized conical surface, the analyzed (or background) radial- and tangential-wind fields are represented by their associated state vectors. We denote by $\mathbf{b}_{\mathrm{r}}$ and $\mathbf{b}_{\tau}$ the state vectors for the background radial and tangential velocities, respectively; and by $\mathbf{a}_{\mathrm{r}}$ and $\mathbf{a}_{\tau}$ the state vectors for the analyzed radial and tangential velocities, respectively. The state vectors for the analyzed radial- and tangential-velocity increments are then given by $\Delta \mathbf{a}_{\mathrm{r}}=\mathbf{a}_{\mathrm{r}}-\mathbf{b}_{\mathrm{r}}$ and $\Delta \mathbf{a}_{\tau}=\mathbf{a}_{\tau}-\mathbf{b}_{\tau}$, respectively. Consider the analysis space composed of two subspaces: one for $\Delta \mathbf{a}_{\mathrm{r}}$ and the other for $\Delta \mathbf{a}_{\tau}$. The state vector for the analyzed incremental vector wind field can be then denoted by $\Delta \mathbf{a}=\left(\Delta \mathbf{a}_{\mathrm{r}}^{\mathrm{T}}, \Delta \mathbf{a}_{\tau}^{\mathrm{T}}\right)^{\mathrm{T}}$. Similarly, the state vector for the background vector wind field can be denoted by $\mathbf{b}=\left(\mathbf{b}_{\mathrm{r}}^{\mathrm{T}}, \mathbf{b}_{\tau}^{\mathrm{T}}\right)^{\mathrm{T}}$.

As in XG03, the radar radial-velocity observation errors are assumed to be Gaussian random with zero mean (unbiased) and to be uncorrelated in space, so the observation error covariance matrix is simply given by $\sigma_{\mathrm{ob}}{ }^{2} \mathbf{I}$ where $\sigma_{\mathrm{ob}}{ }^{2}$ is the observation error variance and $\mathbf{I}$ is the identity matrix in the observation space. The observation errors are not correlated with the background errors either. Under the above assumptions (including those for the background errors mentioned in section 2), the optimal estimate of the true vector wind field is given by the minimizer of the following cost function:

$$
J=\Delta \mathbf{a}^{\mathrm{T}} \mathbf{B}^{-1} \Delta \mathbf{a}+\sigma_{\mathrm{ob}}{ }^{-2}(\mathbf{H} \Delta \mathbf{a}-\mathbf{d})^{\mathrm{T}}(\mathbf{H} \Delta \mathbf{a}-\mathbf{d}),
$$


where $\mathbf{B}$ is the background error covariance matrix, $\mathbf{d}=\mathbf{y}-H(\mathbf{b})$ is the observation innovation vector, $\mathbf{y}$ is the state vector of the radial-velocity observations, $H$ is the observation operator that maps state vectors from the analysis space to the observation space, and $\mathbf{H}$ is the linearization of $H$. As the state vector is formulated in the polar coordinates with $\mathbf{b}=\left(\mathbf{b}_{\mathrm{r}}^{\mathrm{T}}, \mathbf{b}_{\tau}^{\mathrm{T}}\right)^{\mathrm{T}}, H$ is simply a spatial interpolator of $\left(v_{\mathrm{r}}, v_{\tau}\right)$ from the analysis grid to the observation points, it is linear and therefore is the same as $\mathbf{H}$. As the observations are limited to radial velocities only, we have $\mathbf{H} \Delta \mathbf{a}_{\tau}=0$ and thus $\mathbf{H} \Delta \mathbf{a}=\mathbf{H} \Delta \mathbf{a}_{\mathrm{r}}$ in (3.1).

The minimizer of $J$ can be obtained by solving the linear system of algebraic equations derived from $\partial J / \partial \Delta \mathbf{a}=0$. By using the matrix equality in (7B.6) of Jazwinski (1970), the solution of the derived linear system can be cast into the following form:

$$
\Delta \mathbf{a}=\mathbf{B H}^{\mathrm{T}}\left(\mathbf{H B H} \mathbf{H}^{\mathrm{T}}+\sigma_{\mathrm{ob}}{ }^{2} \mathbf{I}\right)^{-1} \mathbf{d} .
$$

As in (4.5) of XG03, the solution in (3.2) is computed in the following two steps:

$$
\begin{aligned}
& \left(\mathbf{H B H}^{\mathrm{T}}+\sigma_{\mathrm{ob}}{ }^{2} \mathbf{I}\right) \mathbf{z}=\mathbf{d}, \\
& \Delta \mathbf{a}=\mathbf{B H}^{\mathrm{T}} \mathbf{z},
\end{aligned}
$$

where $\mathbf{H B H} \mathbf{H}^{\mathrm{T}}$ is a $M \times M$ matrix in the observation space of dimension $M$, and $\mathbf{B H}^{\mathrm{T}}$ is a $2 N \times M$ matrix that represents a linear transformation from the observation space to the analysis space of dimension $2 N$. The analysis space composes of two subspaces of dimension $N$ : one for $\Delta \mathrm{ar}$ and the other for $\Delta \mathbf{a}_{\tau}$. This two-step solution procedure belongs to the statistical interpolation (see Chapters 4-5 of Daley 1991). If the observation space is too large to allow (3.3a) be solved directly, then the approach often used in the statistical interpolation is to partition the observation space into partially overlapping subspaces so that $\mathbf{H B} \mathbf{H}^{\mathrm{T}}$ and $\mathbf{B} \mathbf{H}^{\mathrm{T}}$ become small enough in each subspace. The final solution is not truly global as it is pieced together by local solutions (minimizers in subspaces). In this case, to obtain a truly global solution, it is necessary to use an iterative approach with a descending algorithm to find the minimum of the cost function (Cohn et al. 1998; Daley and Barker 2001). The procedure is commonly referred to as variational which gives the same solution if Eqs. (3.3a,b) are solved directly. In this paper, (3.3a) is solved directly in the observation space without partitioning.

In the limit of vanishing grid spacing (with infinitely high resolution), the analysis field becomes continuous. In this case, by using the similar derivations as presented in section $4 \mathrm{~b}$ of $\mathrm{XG03}$, one can show that the solution is continuous and can be obtained from the following equations:

$$
\begin{aligned}
& \left(\mathbf{C}+\sigma_{\mathrm{ob}}{ }^{2} \mathbf{I}\right) \mathbf{z}=\mathbf{d}, \\
& \Delta \mathrm{v}_{\mathrm{r}}(\mathbf{x})=\sum C_{\mathrm{rr}}\left(\mathbf{x}, \mathbf{x}_{\mathrm{m}}\right) z_{\mathrm{m}}, \\
& \Delta \mathrm{v}_{\tau}(\mathbf{x})=\sum C_{\tau \mathrm{r}}\left(\mathbf{x}, \mathbf{x}_{\mathrm{m}}\right) z_{\mathrm{m}}
\end{aligned}
$$

where $\mathbf{C}$ is a $M \times M$ matrix in the observation space with its $(m, n)$-th component given directly by $C_{\mathrm{rr}}\left(\mathbf{x}_{\mathrm{m}}, \mathbf{x}_{\mathrm{n}}\right), \mathbf{x}_{\mathrm{m}}$ (or $\mathbf{x}_{\mathrm{n}}$ ) denotes the $m$-th (or $n$-th) observation point, the summation $\sum$ is over $m$ from 1 to $M$, and $z_{\mathrm{m}}$ is the $m$-th element of vector $\mathbf{z}$. Here, (3.4a) has the same form as (3.3a) except that $\mathbf{C}$ is directly given by $C_{\mathrm{rr}}\left(\mathbf{x}_{\mathrm{m}}, \mathbf{x}_{\mathrm{n}}\right)$ while $\mathbf{H B H ^ { \mathrm { T } }}$ requires formally an additional step of 
operation for the spatial interpolation represented by $\mathbf{H}$. Thus, (3.4a) is simpler than (3.3a), although both can be solved directly by using the Cholesky decomposition. Since the covariance functions are given analytically in (2.5), $\mathbf{H B H} \mathbf{H}^{\mathrm{T}}$ can also be directly given by $C_{\mathrm{rr}}\left(\mathbf{x}_{\mathrm{m}}, \mathbf{x}_{\mathrm{n}}\right)$ to avoid any accuracy loss caused by the interpolation. This treatment has been commonly used in the standard statistical interpolation. Once the intermediate state vector $\mathbf{z}$ is obtained, the optimal estimate of $\left(\Delta \mathrm{v}_{\mathrm{r}}, \Delta \mathrm{v}_{\tau}\right)$ can be easily obtained from $(3.4 \mathrm{~b}, \mathrm{c})$. This solution is the continuous limit of the discrete solution in $(3.3 \mathrm{~b}, \mathrm{c})$, and as an analysis increment can be easily added back to the background field (only necessary when the background is nonzero) on the same grid used by the background field on the conical surface. This procedure will be used with (3.4) to produce the solutions in the subsequent sections.

If $\mathbf{B}$ is formulated based on the covariance tenser function $\mathbf{C}_{\mathbf{V v}}$ given by (2.1) in the Cartesian coordinates, then $\mathbf{H B H}^{\mathrm{T}}$ will require two additional steps of operation, first for the spatial interpolation to the Cartesian coordinates and then for the projection from the Cartesian coordinate system to the polar coordinate system. This two-step operation will compute the same matrix $\mathbf{H B H}^{\mathrm{T}}$ as in (3.3a) but the computed matrix will be less accurate than that in (3.3a) while the latter is less accurate than that in (3.4a). Thus, as mentioned in the introduction, it is useful to formulate the background error covariance functions in the polar coordinates on the conical surface for the intended application of this paper. The derived error covariance functions will not only simplify the construction of the equation system and related computations but also facilitate direct inspections of the correlation structures, which will be discussed in the next section.

\section{The structure of cross-covariance function as revealed by analysis of a single observation}

The standard approach to examining the effective correlation structure in an analysis scheme is to analyze a single observation. When there is only a single radial-velocity observation at point $\mathbf{x}_{1}$, the observation space becomes one-dimensional. In this case, the matrix $\mathbf{C}+\sigma_{\mathrm{ob}}{ }^{2} \mathbf{I}$, and the vectors $\mathbf{z}$ and $\mathbf{d}$ in (3.4) degenerate into scalars $\sigma^{2}+\sigma_{\mathrm{ob}}{ }^{2}, z$ and $d$, respectively, where $C_{\mathrm{rr}}\left(\mathbf{x}_{1}\right.$, $\left.\mathbf{x}_{1}\right)=\sigma^{2}$ is used according to (2.5a). The solution of (3.4a) is simply $z=d /\left(\sigma^{2}+\sigma_{\mathrm{ob}}{ }^{2}\right)$. The solutions of (3.4b) and (3.4c) yield

$$
\text { and } \quad \begin{aligned}
& \Delta v_{\mathrm{r}}(\mathbf{x})=C_{\mathrm{rr}}\left(\mathbf{x}, \mathbf{x}_{1}\right) d /\left(\sigma^{2}+\sigma_{\mathrm{ob}}{ }^{2}\right), \\
& \Delta v_{\tau}(\mathbf{x})=C_{\tau \mathrm{r}}\left(\mathbf{x}, \mathbf{x}_{1}\right) d /\left(\sigma^{2}+\sigma_{\mathrm{ob}}{ }^{2}\right),
\end{aligned}
$$

for the radial-velocity and tangential-velocity increment fields, respectively, with $\mathbf{x}$ being discretized onto the analysis grid. These two solutions are identical to the covariance functions $C_{\mathrm{rr}}\left(\mathbf{x}, \mathbf{x}_{1}\right)$ and $C_{\tau \mathrm{r}}\left(\mathbf{x}, \mathbf{x}_{1}\right)$, respectively, except for the factor of $d /\left(\sigma^{2}+\sigma_{\mathrm{ob}}{ }^{2}\right)$. Thus, the two correlation functions, $C_{\mathrm{rr}}\left(\mathbf{x}, \mathbf{x}_{1}\right) / \sigma^{2}$ and $C_{\tau \mathrm{r}}\left(\mathbf{x}, \mathbf{x}_{1}\right) / \sigma^{2}$, can be viewed as the radial- and tangentialcomponent fields of the vector increment field produced by a single radial-velocity observation with an innovation of $d=1+\sigma_{\mathrm{ob}} / \sigma^{2}$ at point $\mathbf{x}_{1}$. The structure of the auto-correlation function, $C_{\mathrm{rr}}\left(\mathbf{x}, \mathbf{x}_{1}\right) / \sigma^{2}$, was analyzed in section 5 of XG03. Here, we only need to analyze the structure of the cross-correlation function, $C_{\tau \mathrm{r}}\left(\mathbf{x}, \mathbf{x}_{1}\right) / \sigma^{2}$.

As in the first case selected in XG03, $a=0$ and $b=1$ can be selected for simplicity in (2.4). In this case, the rotational and divergent parts of the background wind error are assumed to have the same variance $\left(\sigma_{\mathrm{rot}^{2}}{ }^{2}=\sigma_{\mathrm{div}}{ }^{2}=\sigma^{2}\right)$ and the same decorrelation length scale $\left(L_{\mathrm{rot}}=L_{\mathrm{div}}=L\right)$, as this leads to $a=0$ and $b=1$ [see (3.5)-(3.7) of XG03]. Substituting these parameter values into (2.5) and then into (4.1) gives 


$$
\begin{aligned}
& \Delta v_{\mathrm{r}}(\mathbf{x})=C_{\mathrm{rr}}\left(\mathbf{x}, \mathbf{x}_{1}\right) / \sigma^{2}=\exp \left[-r^{2} /\left(2 L^{2}\right)\right] \cos \left(\beta_{1}-\beta\right) \\
& \Delta v_{\tau}(\mathbf{x})=C_{\tau \mathrm{r}}\left(\mathbf{x}, \mathbf{x}_{1}\right) / \sigma^{2}=\exp \left[-r^{2} /\left(2 L^{2}\right)\right] \sin \left(\beta_{1}-\beta\right)
\end{aligned}
$$

where $r=\left|\mathbf{x}-\mathbf{x}_{1}\right|=\left[\left(x-x_{1}\right)^{2}+\left(y-y_{1}\right)^{2}\right]^{1 / 2}$ and $\beta=\tan ^{-1}(y / x)$. It is easy to see $C_{\tau \mathrm{r}}\left(\mathbf{x}, \mathbf{x}_{1}\right)=-$ $C_{\mathrm{r} \tau}\left(\mathbf{x}, \mathbf{x}_{1}\right)=C_{\mathrm{r} \tau}\left(\mathbf{x}_{1}, \mathbf{x}\right)$. An example of $C_{\tau \mathrm{r}}\left(\mathbf{x}, \mathbf{x}_{1}\right) / \sigma^{2}$ or $\Delta v_{\tau}(\mathbf{x})$ in (4.2b) is plotted in Fig. 1a for $\mathbf{x}_{1}$ $=\left(x_{1}, y_{1}\right)=(-15,-15) \mathrm{km}$ and $L=30 \mathrm{~km}$, where the coordinate system is radar-relative (with the origin at the radar) and the plotting domain covers an area of $120 \times 120 \mathrm{~km}^{2}$ centered at the radar. It is easy to see from (4.2b) and Fig. 1a that $\sin \left(\beta_{1}-\beta\right)=\sin (\pi / 4-\beta)$ is anti-symmetric with respect to the southwest-northeast diagonal line defined by $y=x$, as is $\Delta v_{\tau}(\mathbf{x})$. Along $y=x$, we have $\sin \left(\beta_{1}-\beta\right)=0$ and thus $\Delta v_{\tau}(\mathbf{x})=C_{\tau \mathrm{r}}\left(\mathbf{x}, \mathbf{x}_{1}\right) / \sigma^{2}=0$ as shown by the straight zero contour along the southwest-northeast diagonal in Fig. 1a. On the upper-left (or lower-right) side of this diagonal line, $\Delta v_{\tau}(\mathbf{x})$ is positive (or negative). Note that the observed radial velocity (with $d=1$ $+\sigma_{\mathrm{ob}} 2 / \sigma^{2}>0$ ) at $\mathbf{x}_{1}$ has a positive (or negative) projection in the tangential direction at point $\mathbf{x}$ if this point is on the upper-left (or lower-right) side of the diagonal line. This explains why the tangential-velocity increment $\Delta v_{\tau}(\mathbf{x})$ inferred from the single radial-velocity observation at $\mathbf{x}_{1}$ is positive (or negative) on the upper-left (or lower-right) side of the diagonal line.

If the decorrelation length scale $L$ becomes infinitely large, then $\Delta v_{\mathrm{r}}(\mathbf{x})=\cos \left(\beta-\beta_{1}\right)$ and $\Delta v_{\tau}(\mathbf{x})=\sin \left(\beta-\beta_{1}\right)$ according to (4.2) or (2.5). In this case, the vector wind field inferred from the single radial-velocity observation at $\mathbf{x}_{1}$ is uniformly northeastward (parallel to vector $\mathbf{x}_{1}$ ), while $\beta_{1}-\beta$, the angle of vector $\mathbf{x}_{1}$ with respect to vector $\mathbf{x}$ (positive for a counterclockwise rotation from $\mathbf{x}$ to $\mathbf{x}_{1}$ ), is also the angle of the inferred vector wind (parallel to vector $\mathbf{x}_{1}$ ) with respect to vector $\mathbf{x}$. For a finite $L$ (such as $L=30 \mathrm{~km}$ in Fig. 1a), the amplitude of the above uniform vector velocity field (inferred with infinitely large $L$ ) is masked by $\exp \left[-r^{2} /\left(2 L^{2}\right)\right]$. This masked vector velocity field is plotted in Fig. 1b. It is easy to verify that the projections of the masked vector velocity onto the radial and tangential directions at a given point $\mathbf{x}$ gives exactly the radial component $\Delta v_{\mathrm{r}}(\mathbf{x})$ in (4.2a) and tangential component $\Delta v_{\tau}(\mathbf{x})$ in (4.2b), respectively.

If the rotational and divergent parts of the background wind error do not have the same variance, then $a \neq 0$ and the full function forms of $C_{\mathrm{rr}}\left(\mathbf{x}, \mathbf{x}_{1}\right)$ and $C_{\tau \mathrm{r}}\left(\mathbf{x}, \mathbf{x}_{1}\right)$ need to used in (4.2) with $\left(\mathbf{x}_{1}, \mathbf{x}_{2}\right), \Delta \beta_{1}$ and $\Delta \beta_{2}$ in (2.5) replaced by $\left(\mathbf{x}, \mathbf{x}_{1}\right), \Delta \beta$ and $\Delta \beta_{1}$, respectively. Note that $\Delta \beta_{1}$ $\Delta \beta=\beta_{1}-\beta$ is the angle of vector $\mathbf{x}_{1}$ with respect to vector $\mathbf{x}$, while $\Delta \beta_{1}=\alpha-\beta_{1}$ and $\Delta \beta=\alpha-\beta$ are the angles of vector $\mathbf{x}-\mathbf{x}_{1}$ with respect to vectors $\mathbf{x}_{1}$ and $\mathbf{x}$, respectively (see Fig. 1 in XG03 but with $\mathbf{x}_{2}$ viewed as $\mathbf{x}$ ). Thus, for a mirror reflection of $\mathbf{x}$ with respect to vector $\mathbf{x}_{1}, \Delta \beta_{1}$ and $\Delta \beta$ change their signs but not their absolute values. For this mirror reflection, $\cos \left(\Delta \beta_{1}-\Delta \beta\right)$ and $\cos \left(\Delta \beta_{1}+\Delta \beta\right)$ are invariant while $\sin \left(\Delta \beta_{1}-\Delta \beta\right)$ and $\sin \left(\Delta \beta_{1}+\Delta \beta\right)$ change their signs only. This implies that the auto-correlation function is always symmetric (see Figs. 3a-b of XG03) and the cross-correlation function is always anti-symmetric (Fig. 1a) with respect to the straight line along vector $\mathbf{x}_{1}$. The observed radial wind at $\mathbf{x}_{1}$ always has zero projection in the tangential direction at point $\mathbf{x}$ if this point is on the straight line along vector $\mathbf{x}_{1}$. This explains why the zero contour of the cross-correlation function is always along vector $\mathbf{x}_{1}$, as shown by the simple example in Fig. 1a. This is also simply the limiting case of $\Delta \beta_{1}$ and $\Delta \beta_{2} \rightarrow 0$ or $\pi$, as discussed for (2.3) in section 2. 


\section{Analysis experiments with simulated and real radar observations}

a. Analyses of simulated radar observations with incomplete coverage for an idealized flow

Radar radial-velocity observations are dense but the coverage is often incomplete within the analysis domain, partly because there are usually only available in precipitation regions. The impact of data void areas on the radial-velocity analysis was examined by an idealized example in XG03. Here, we examine the impact of data void areas on the tangential wind analysis by a similar idealized example. In this idealized case, the true wind field is assumed to be uniformly northeastward with $U=10 \mathrm{~m} \mathrm{~s}^{-1}$ and $V=10 \mathrm{~m} \mathrm{~s}^{-1}$, so the true radial and tangential velocities are given by $U \cos \beta+V \sin \beta$ and $-U \sin \beta+V \cos \beta$, respectively, as plotted in Fig. 2a and Fig. 2b. The simulated radial-velocity observations are generated by adding random errors with $\sigma_{\mathrm{ob}}=1 \mathrm{~m} \mathrm{~s}^{-1}$ to the true field on a polar grid centered at the radar with resolutions of $1 \mathrm{~km}$ in the radial direction and $2^{\circ}$ in the azimuthal direction (to mimic thinned radar radial-velocity observations); but the observations cover only the left half of the domain. As shown by the shaded area in Fig. 2a, observations within $10 \mathrm{~km}$ of the radar are also removed to mimic the removal of ground clutter in the real situation. The analysis domain is set to $120 \times 120 \mathrm{~km}^{2}$ centered at the radar.

For the type of mesoscale wind analysis that we desire, operational numerical weather prediction models rarely provide reliable enough prediction for use as the analysis background; the forecast low-level winds often contain significant biases and/or large phase errors. In the absence of a bias-free or bias-corrected background with reliable background error estimates, a background with zero mean but non-zero error covariance can be the best alternative. In this case, the analysis can still be interpreted as a Bayes estimate of the true field based on the observations but the true wind field is assumed to be Gaussian random with zero mean and nonzero covariance (see section 1.5 of Wahba 1990 and related remarks in the last two paragraphs of Xu 2005).

Based on the above considerations, here we assume that the background field is zero with the standard deviation of the background error given by $\sigma=10 \mathrm{~m} \mathrm{~s}^{-1}$. The parameter settings for the correlation functions are $a=0$ and $b=1$ (corresponding to $\sigma_{\mathrm{div}} / \sigma_{\mathrm{rot}}=1$ and $L_{\mathrm{div}} / L_{\mathrm{rot}}=1$, as explained at the end of section 2) with different values of $L(\geq 30 \mathrm{~km})$. Since zero background is used, the actual background error is equal to the true wind. In this case, the wind field in Fig. 2 is considered to be a statistical sample of the true random field that is spatially uniform, so the decorrelation length scale is infinitely large. With this setting $(L=\infty)$, the analyzed vector wind field almost exactly recovers the true wind field in Fig. 2, and the root-mean-square (rms) differences between the analyzed and true winds are 0.0357 and $0.0358 \mathrm{~m} \mathrm{~s}^{-1}$ for the radial- and tangential-component fields, respectively. This nearly perfect result is not surprising because the error covariance structure is precisely known and specified (with $L=\infty$ ) for this idealized case.

For the purpose of comparison, we also performed an analysis by using the traditional VAD method (Browning and Wexler 1968). In contrast, the VAD analysis is far less accurate; the rms differences between the VAD and true winds are 0.27 and $0.12 \mathrm{~m} \mathrm{~s}^{-1}$ for the radial and tangential components, respectively. Thus, if the decorrelation length scale is precisely set for this idealized uniform-wind case, then the current method can be more accurate than the VAD method in the presence of random observation errors, even when the horizontally-uniform-flow assumption of $\mathrm{VAD}$ is valid. This is mainly because the current method uses all the observations on the conical surface to simultaneously estimate the vector winds and thus can filter observation noise more effectively than the VAD analysis.

Now consider that the decorrelation length scale, $L$, is unknown but subjectively set to, for example, $60 \mathrm{~km}$ or even smaller. The radial- and tangential-component fields obtained with $L=$ 
$60 \mathrm{~km}$ are shown in Figs. 3a and 3b, respectively. As shown, the analyzed radial-velocity field in Fig. 3a is very close to the true one given in Fig. 2a in the data dense area in the left half of the domain, except for the northeast corner area where the radial wind is underestimated due to the lack of observation. The analyzed tangential-wind field in Fig. $3 \mathrm{~b}$ is also very close to the true one in Fig. $2 b$ in the immediate vicinity of the radar and in the northeast and southwest sectors. However, the tangential winds are underestimated by the analysis in the northwest and southeast corner areas and the largest difference is seen at the southeast corner point. Unlike the true tangential-wind field, the analyzed tangential-wind field is not exactly anti-symmetric with respect to the southwest-northeast diagonal line. This deviation from the anti-symmetry is caused by the asymmetry in the data coverage. The underestimated tangential winds in the northwest and southeast corner areas are mainly due to the finite decorrelation length scale specified $(L=$ $60 \mathrm{~km}$ ). The situation becomes slightly worse as the decorrelation length scale is reduced further to $30 \mathrm{~km}$ (not shown, see Fig. 5 of XG03 for the analysis of radial-velocity).

From the structure analysis of the cross-covariance function in section 4, we know that no tangential-wind information can be obtained for a radial-velocity observation at the observation point and this is indicated simply by $C_{\tau \mathrm{r}}\left(\mathbf{x}_{1}, \mathbf{x}_{1}\right)=0$ according to $(2.5 \mathrm{~b})$. Thus, a piece of information on the tangential wind at a given point $\mathbf{x}$ can be inferred only from a radial-velocity observation at a neighboring point, say, $\mathbf{x}_{1}$, where the observed radial wind has non-zero projection on the tangential direction at the given point $\mathbf{x}$. However, the inferred information diminishes significantly as the distance between the two points (measured by $r / L$ where $r=\mid \mathbf{x}$ $\left.\mathbf{x}_{1} \mid\right)$ increases beyond the decorrelation length scale or as the angle of vector $\mathbf{x}_{1}$ with respect to vector $\mathbf{x}$ (measured by $\Delta \beta_{1}-\Delta \beta=\beta_{1}-\beta$ ) diminishes. These properties of statistical interpretation are tied up with the mathematical form of the cross-correlation function in (4.2b). Based on these properties, we can easily understand why the true tangential winds are captured by the analysis reasonably well in the immediate vicinity of the radar and in the northeast and southwest sectors even though the decorrelation length scale is subjectively set to a finite value in the mesoscale range (rather than accurately set to infinity).

\section{b. Experiments with idealized convergent and circular flows}

This subsection presents two additional idealized-flow experiments to examine the capability and limitations of our method for our intended application, that is, to estimate the mesoscale environmental vector wind field on the conical surface of low-elevation radar scans. The first experiment considers an idealized convergent flow. As shown in Fig. 4a, this idealized flow converges sharply along a southwest-northeast oriented straight line located to the northwest of the radar (at the domain center). The true vector winds have constant values of ( $U$, $V)=(0,20)$ and $(15,-15) \mathrm{m} \mathrm{s}^{-1}$ on the southeast and northwest sides, respectively, of the convergence line. The radial-velocity observations are generated from the true winds by adding random errors with $\sigma_{\mathrm{ob}}=1 \mathrm{~m} \mathrm{~s}^{-1}$ in the same way as in the previous subsection. The parameter settings for the background error covariance are also the same as in the previous subsection, and the analysis is performed with a decorrelation length scale of $30 \mathrm{~km}$. This setting of $L$ is rather subjective but is roughly consistent with the mesoscale feature of the concerned flow field (which is also the background error field for a zero-mean background).

Strictly speaking, especially for real-data applications, the decorrelation length scale needs to be estimated statistically together with other covariance parameters $(\sigma, a$ and $b)$ from, e.g., time series of radar radial-velocity innovation (observation minus background) fields. The estimation can be done in the same way as in Xu et al. $(2003,2006)$ except that the background covariance functions are modeled by (2.4) here (instead of the truncated spectral expansions used in the 
cited studies); thus the above parameters can be estimated by fitting (2.5a) to radar radialvelocity innovation covariance data. This problem deserves further studies but the topic is beyond the scope of the current paper.

With the decorrelation length scale, $L$, set to $30 \mathrm{~km}$, the analyzed vector wind field is shown in Fig. 4b. As shown, the method is able to estimate the convergent winds on the mesoscale but cannot resolve the small-scale sharp convergence line found in the true field. Clearly, the method is limited to the mesoscale as the decorrelation length scale is set to $30 \mathrm{~km}$ in the mesoscale range. For the reason explained at the end of the previous subsection, the decorrelation length scale cannot be set to too small a value (with respect to the radial distance from the radar) and thus the method is not expected to be able to retrieve or resolve small-scale features. Regardless of this limitation, the method is satisfactory in capturing the mesoscale convergent winds in this case. The rms differences between the analyzed and true winds are 4.3 and $6.8 \mathrm{~m} \mathrm{~s}^{-1}$ for the radial- and tangential-component fields, respectively, and the differences are caused mainly by the discrepancies (not shown) within $\pm 20 \mathrm{~km}$ along the front. When the VAD method is used, the rms differences increase to 12.5 and $12 \mathrm{~m} \mathrm{~s}^{-1}$, respectively, and the VAD wind field (not shown) bears little resemblance to the true convergent flow. Our method is clearly superior to VAD in this case.

The second experiment considers an idealized circular flow associated with an axisymmetric vortex centered at the northeast corner $(x=60 \mathrm{~km}$ and $y=60 \mathrm{~km})$ of the domain as shown in Fig. 5a. The flow is described by a modified Rankine vortex (Brown et al. 2002): $V=V_{\max }(r / R)^{n}$, where $V$ is the rotational velocity at radius $r$ (from the vortex center), $V_{\max }=30 \mathrm{~m} \mathrm{~s}^{-1}$ is the maximum velocity at $r=R=30 \mathrm{~km}$, and $n=1$ for $r \leq R$ and $n=-0.6$ for $r>R$. The radialvelocity observations are again generated by adding random errors with $\sigma_{\mathrm{ob}}=1 \mathrm{~m} \mathrm{~s}^{-1}$. The parameter settings for the background error covariance are the same as in the previous subsection, and again the analysis is performed with the decorrelation length scale set to $30 \mathrm{~km}$.

The analyzed vector wind field is shown in Fig. 5b; clearly it bears a close resemblance to the true flow pattern in Fig. 5a. The rms differences between the analyzed and true winds are 1.8 and $4.7 \mathrm{~m} \mathrm{~s}^{-1}$ for the radial- and tangential-component fields, respectively. When the VAD analysis is used, the rms differences increase to 5.7 and $5.6 \mathrm{~m} \mathrm{~s}^{-1}$, respectively. As shown in Figs. $5 \mathrm{a}$ and $5 \mathrm{~b}$, although the true winds are perpendicular to the radar beam along the southwest-northeast diagonal line, the circular flow pattern can be partially captured by the analysis of the current method but not by the VAD analysis (not shown). However, if the vortex moves from the northeast corner into the domain, then the reversed wind on the far side of the vortex center away from the radar will not be captured by our method. This limitation is understandable based on the reasons explained at the end of previous subsection. Such a limitation is tolerable because the current method only needs to analyze the mesoscale environmental flow while small-scale vortices are expected to be retrieved by the nested $2 \mathrm{dSA}$, as discussed in the introduction.

\section{c. Analyses of real radar observations}

The level-II radial velocity observations collected by the Oklahoma City WSR-88D (KTLX) radar on 16 June 2002 for a surface cold front over the state of Oklahoma are analyzed to demonstrate the performance of our method. The data have a radial resolution of $250 \mathrm{~m}$ while the azimuthal resolution is about 1 degree. The dealiased radial velocities on the conical surface of 0.5 degree elevation at 0040 UTC are plotted in Fig. 6 for an area of $120 \times 120 \mathrm{~km}^{2}$ centered at the radar. As can be seen, the radial velocity observations are negative in the northwest corner area and in the southwest sector of the analysis domain. Between these two areas, there is hardly 
any data available. The observed negative radial winds in those two areas suggest that the vector wind field should converge in this data void zone. The existence of such a convergence zone is consistent with the presence of a surface cold front in the operational surface analysis (not shown). In association with this cold front, there was a precipitation band stretching in the southwestward-northeastward direction and moving southeastwards. The dealiased radial velocity data are thinned by selecting one observation every four gates and from every other radial. The thinned observations therefore have the reduced resolutions of $1 \mathrm{~km}$ in the radial direction and $2^{\mathrm{O}}$ in the azimuthal direction. In this space of thinned observations, the radialvelocity observation errors can be safely assumed to be uncorrelated (Xu et al. 2003, 2006).

The analysis is again performed with a zero background. The background error standard deviation is again set to $\sigma=10 \mathrm{~m} \mathrm{~s}^{-1}$. The results are shown in Figs. 7a-c. As shown in Fig. 7c, the analyzed vector winds converge along a curved zone stretching along the line of $\mathrm{y}=5 \mathrm{~km}$ near the west boundary that then turns northeastward along the diagonal line of $x=y$. As mentioned earlier, the actual existence of this wind convergence zone is confirmed by the presence at the same location of a cold front in the operational surface analysis (not shown). In the northeast corner area, the analyzed vector winds are largely southwesterly, which is somewhat similar to that in the idealized case in section $5 \mathrm{a}$ and the convergent flow case in section $5 \mathrm{~b}$. Because of this, the analyzed radial and tangential winds in the northeast corner area in Figs. $7 \mathrm{a}$ and $7 \mathrm{~b}$ have similar patterns as those in Figs. 3a and 3b, respectively. However, since the vector wind field is neither uniform nor unidirectional as estimated in Fig. 7c, the overall patterns of the analyzed radial and tangential winds in Figs. $7 \mathrm{a}$ and $7 \mathrm{~b}$ are quite different from those for the idealized case in Figs. $3 a$ and $3 b$. These results suggest that the method can be used to analyze mesocale non-uniform and non-unidirectional vector winds from radar radial-velocity observations and thus to serve the purpose discussed in the introduction.

\section{Conclusions}

In this paper, the cross-covariance function between the background radial (along the radar beam) and tangential (perpendicular to the beam) wind errors is derived. The derived crosscovariance function is combined with the auto-covariance function derived in XG03 for the background radial-velocity errors to form a complete set of covariance functions. This set of covariance functions is formulated analytically, as in XG03, by downscaling the error covariance functions estimated for the background winds at $850 \mathrm{hPa}$ in $\mathrm{Xu}$ and Wei (2001), while the analytical function forms allow flexible specifications of error variances and decorrelation length scales for the rotational and divergent parts of background wind error statistics. This set of analytical covariance functions can be used within the statistical interpolation framework for mesoscale vector wind analyses from radar-observed radial velocities on a conical surface of low-elevation radar scans.

The structures of the auto- and cross-correlation functions are examined in terms of the influence of a single radial-velocity observation on the analyzed radial- and tangential-velocity increment fields, respectively. In particular, it is shown how the geometric features in the autocorrelation and cross-correlation functions are related to the analyzed vector wind increment field when analyzing a single radial-velocity observation. When no good estimate of the background wind is available and a zero background is assumed, the derived covariance functions can be used as influence functions for the vector-wind analysis. As in XG03, the choice of a zero background is a practical treatment in the absence of a better background field; in such a case, the error of the background is equal to the true wind field itself and the background error covariance is just the covariance of the true wind perturbations with respect to 
the statistical mean of the true total wind. The utility of the derived covariance functions is demonstrated in this paper by numerical experiments using both simulated data from idealized flows and real radar observations. In particular, the results obtained with real radar observations show that the derived covariance functions can be used to analyze mesoscale non-uniform and non-unidirectional vector winds, on the conical surface of radar scans at a low elevation angle (see Figs. 6-7).

The derived covariance functions can be also used for a vector wind analysis in the onedimensional periodic space along a circle of a fixed range on the conical surface of radar scans at any elevation angle. When the decorrelation length scale is set to be infinitely large, this onedimensional analysis produces essentially the same results as the VAD analysis. In this sense, the method developed in this paper is an extension of the traditional VAD wind analysis from a onedimensional to a two-dimensional domain and from the application to uniform and unidirectional vector wind fields (assumed by the VAD method) to non-uniform and non-unidirectional vector wind fields. In principle, the one-dimensional analysis (as an extension of the VAD analysis) may be applied to radial-velocity observations at any elevation angle but this application is not examined in this paper. With the current method, the two-dimensional analysis is limited to radial-velocity observations at a low-elevation angle. To apply the analysis to radar scans at a relatively high elevation angle $\left(>5^{\circ}\right)$, the covariance functions derived in this paper should be extended to consider background wind error correlation in the vertical also. This problem is under investigation.

The statistical interpolation used in this paper cannot be truly optimal unless the observation and background error statistics are accurately represented for the concerned flow. It is thus desirable and important to estimate these error statistics accurately in an objective way. This problem was examined in $\mathrm{Xu}$ et al. (2003, 2006), and the results show that these error statistics can be estimated objectively from time series of radar radial-velocity innovation (observation minus background) fields. As explained in section $5 \mathrm{~b}$, the covariance parameters considered in this paper can be estimated in the same way as in above cited studies except that the background covariance functions are modeled in analytical forms as given in (2.4) of this paper. The estimated error covariance parameters can be then directly used by the method presented in this paper. Likewise, the method presented in this paper can be modified to use the spectral-form background covariance functions used for the error covariance estimation in above cited studies. Thus, the vector wind analysis can be combined with error covariance estimation in different ways to produce the desired mesoscale environmental wind analysis for our intended purpose. Developing such an approach that combines the wind analysis with error covariance estimation and yet still can be implemented efficiently in real time within our storm targeted radar wind retrieval system remains a challenging task and requires continued research efforts.

Acknowledgments. The authors are thankful to Dr. William Martin and the anonymous reviewers for their comments and suggestions that improved the presentation of the results. This research was primarily supported by ONR Grants N000140310822 and N000140410312 at the University of Oklahoma. Q. Xu and S. Liu were also supported by FAA contract IA\# DTFA03-01-X-9007 and by the NOAA HPCC program. M. Xue was also supported by NSF grants ATM-0530814, ATM-0331756, ATM-0331594 and EEC-0313747. The authors also acknowledge the computational resources provided by the OU Supercomputing Center for Education \& Research (OSCER) at the University of Oklahoma. Funding was also provided to CIMMS by NOAA/Office of Oceanic and Atmospheric Research under NOAA-University of Oklahoma Cooperative Agreement \#NA17RJ1227, U.S. Department of Commerce. 


\section{REFERENCE}

Barker, E. H. 1992: Design of the Navy's multivariate optimum interpolation analysis system. Wea. Forecasting, 7, 220-231.

Brown, R. A., Wood V. T. and Sirmans. D. 2002: Improved tornado detection using simulated and actual WSR-88D data with enhanced resolution. J. Atmos. Ocean. Tech., 19, 1759-1771.

Browning, K. A. and Wexler, R. 1968: The determination of kinematic properties of a wind field using Doppler radar, J. Appl. Meteor., 7, 105-113.

Cohn, S., da Silva, A., Guo, J., Sienkiewicz, M. and Lamich, D. 1998: Assessing the effects of data selection with the DAO Physical-Space Statistical Analysis System. Mon. Wea. Rev., 126, 2913-2926.

Daley, R. 1991: Atmospheric Data Analysis. Cambridge University Press, 457 pp.

Daley, R. and Barker, E. 2001: NAVDAS: Formulation and Diagnostics. Mon. Wea. Rev., 129, 869-883.

Gal-Chen, T. 1982: Errors in fixed and moving frame of references: Applications for conventional and Doppler radar analysis. J. Atmos. Sci., 39, 2279-2300.

Gao, J., Xue, M., Shapiro, A., Xu, Q. and Droegemeier, K. K. 2001: Three-dimensional simple adjoint velocity retrievals from single Doppler radar, J. Atmos. \& Oceanic Technology, 2638.

Gao, J., Brewster, K. and Xue, M. 2006: A comparison of the radar ray path equations and approximations for use in radar data assimilation. Adv. Atmos. Sci., 23, 190-198.

Jazwinski, A. H. 1970: Stochastic Processes and Filtering Theory. Academic Press, 376 pp.

Lhermitte, R.M. and Atlas, D. 1961: Precipitation motion by pulse Doppler, Preprints, 9th Weather Radar Conference, Kansas City, AMS, 218-223.

Liou, Y.-C. 1999: Single radar recovery of cross-beam wind components using a modified moving frame of reference technique. J. Atmos. Oceanic Technol., 16, 1003-1016.

Liou, Y.-C. and Luo. I.-S. 2001: An investigation of the moving-frame single-Doppler wind retrieval technique using Taiwan area mesoscale experiment low-level data. J. Appl. Meteorology, 40, 1900-1917.

Laroche, S. and Zawadzki, I. 1994: A variational analysis method for retrieval of threedimensional wind field from single-Doppler radar data. J. Atmos. Sci., 51, 2664-2682.

Liu, S., Qiu, C., Xu, Q. and Zhang, P. 2004: An improved time interpolation for threedimensional Doppler wind analysis. J. Appl. Meteor., 43, 1379-1391.

Lorenc, A. 1981: A global three-dimensional multivariate statistical analysis system. Mon. Wea. Rev., 109, 701-721.

Parrish, D. F. and Derber, J. C. 1992: The National Meteorological Center's spectral statisticalinterpolation analysis system. Mon. Wea. Rev., 120, 1747-1763.

Panchev, S. 1971: Random functions and turbulence. Pergamon Press, $444 \mathrm{pp}$.

Qiu, C. and Xu, Q. 1992: A simple adjoint method of wind analysis for single-Doppler data. $J$. Atmos. \& Oceanic Technology, 9, 588-598.

Qiu, C. and $\mathrm{Xu}, \mathrm{Q}$. 1996: Least-square retrieval of microburst winds from single-Doppler radar data. Mon. Wea. Rev., 124, 1132-1144.

Shapiro, A., Ellis, S. and Shaw, J. 1995: Single-Doppler velocity retrievals with Phoenix II data: clear air and microburst wind retrievals in the planetary boundary layer. J. Atmos. Sci., 52, $1265-1287$.

Sun, J. and Crook, N. A. 1994: Wind and thermodynamic retrieval from single-Doppler measurements of a gust front observed during Phoenix II. Mon. Wea. Rev., 122, 1075-1091. 
Sun, J. and Crook, N. A. 2001: Real-time low-level wind and temperature analysis using single WSR-88D data. Weather and Forecasting. 16, 117-132.

Wahba, G. 1990: Spline Models for Observation Data. Society for Industrial and Applied Mathematics. Capital City Press, 169 pp.

Weygandt, S. S., Shapiro, A. and Droegemeier, K. K. 2002: Retrieval of initial forecast fields from single-Doppler observations of a supercell thunderstorm. Part I: Single-Doppler velocity retrieval. Mon. Wea. Rev., 130, 433-453.

Xu, Q. 2005: Representations of inverse covariances by differential operators. Adv. Atmos. Sci., 22, 181-198.

Xu, Q. and Gong, J. 2003: Background error covariance function for Doppler radar radial-wind analysis. Quart. J. Roy. Meteor. Soc., 129, 1703-1720.

Xu, Q., Gu, H. and Qiu, C. 2001a: Simple adjoint retrievals of wet-microburst winds and gustfront winds from single-Doppler radar data. J. Appl. Meteor., 40, 1485-1499.

Xu, Q., Qiu, C., Gu, H. and Yu, J. 1995: Simple adjoint retrievals of microburst winds from single-Doppler radar data. Mon. Wea. Rev., 123, 1822-1833.

Xu, Q., Gu, H. and Yang, S. 2001b: Simple adjoint method for three-dimensional wind retrievals from single-Doppler radar. Quart. J. Roy. Meteor. Soc., 127, 1053-1067.

Xu, Q., Nai, K. and Wei, L. 2006: An innovation method for estimating radar radial-velocity observation error and background wind error covariances. Quart. J. Roy. Meteor. Soc. (accepted)

Xu, Q., Qiu, C. and Yu, J. 1994: Adjoint-method retrievals of low-altitude wind fields from single-Doppler reflectivity measured during Phoenix II. J. Atmos. Oceanic Technol., 11, 275-288.

Xu, Q., Wang, L. and Nai, K. 2003: Error covariance estimation for Doppler wind data assimilation. Preprints, 31th Conference on Radar Meteorology, 6-12 August 2003, Seattle, Washington, Amer. Meteor. Soc., 108-109.

Xu, Q. and Wei, L. 2001: Estimation of three-dimensional error covariances. Part II: Analysis of wind innovation vectors. Mon. Wea. Rev. 129, 2939-2954.

Zhang, P., Liu, S., Xu, Q. and Song, L. 2005: Storm targeted radar wind retrieval system. 32nd Conference on Radar Meteorology, 24-29 October 2005, Albuquerque, New Mexico, Amer. Meteor. Soc., P8R1, Conference CD.

Zhang, J. and Gal-Chen, T. 1996: Single-Doppler wind retrieval in the moving frame of Reference. J. Atmos. Sci., 53, 2609-2623. 

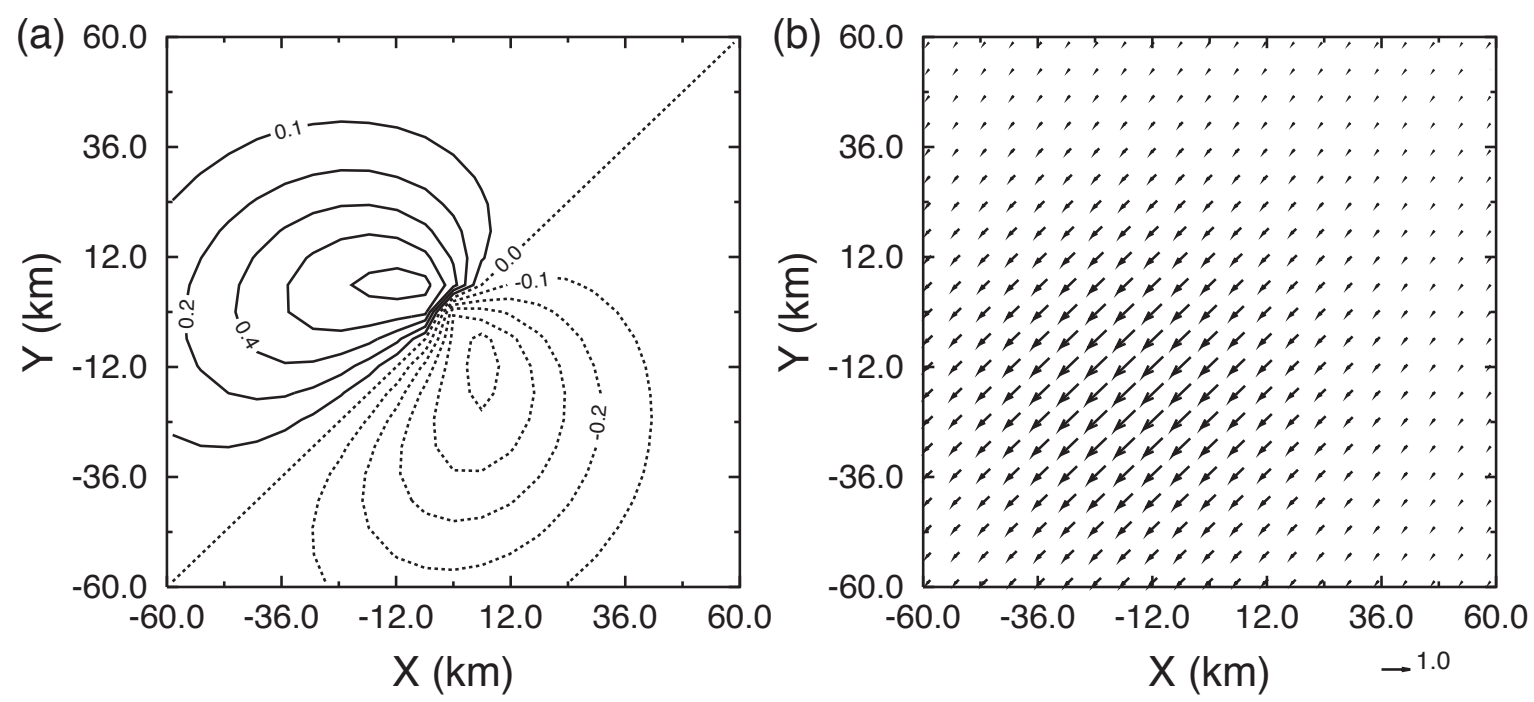

Fig. 1. Cross-correlation function (a), and masked vector velocity field composed of the auto-correlation function and cross-correlation function (b). The two correlation functions are given by (4.2a) and (4.2b) with $\mathbf{x}_{1}=\left(x_{1}, y_{1}\right)=(-15,-15) \mathrm{km}$ and $L=30 \mathrm{~km}$. In panel (a), contours are plotted every 0.1 with solid for non-negative and dotted for negative values. 

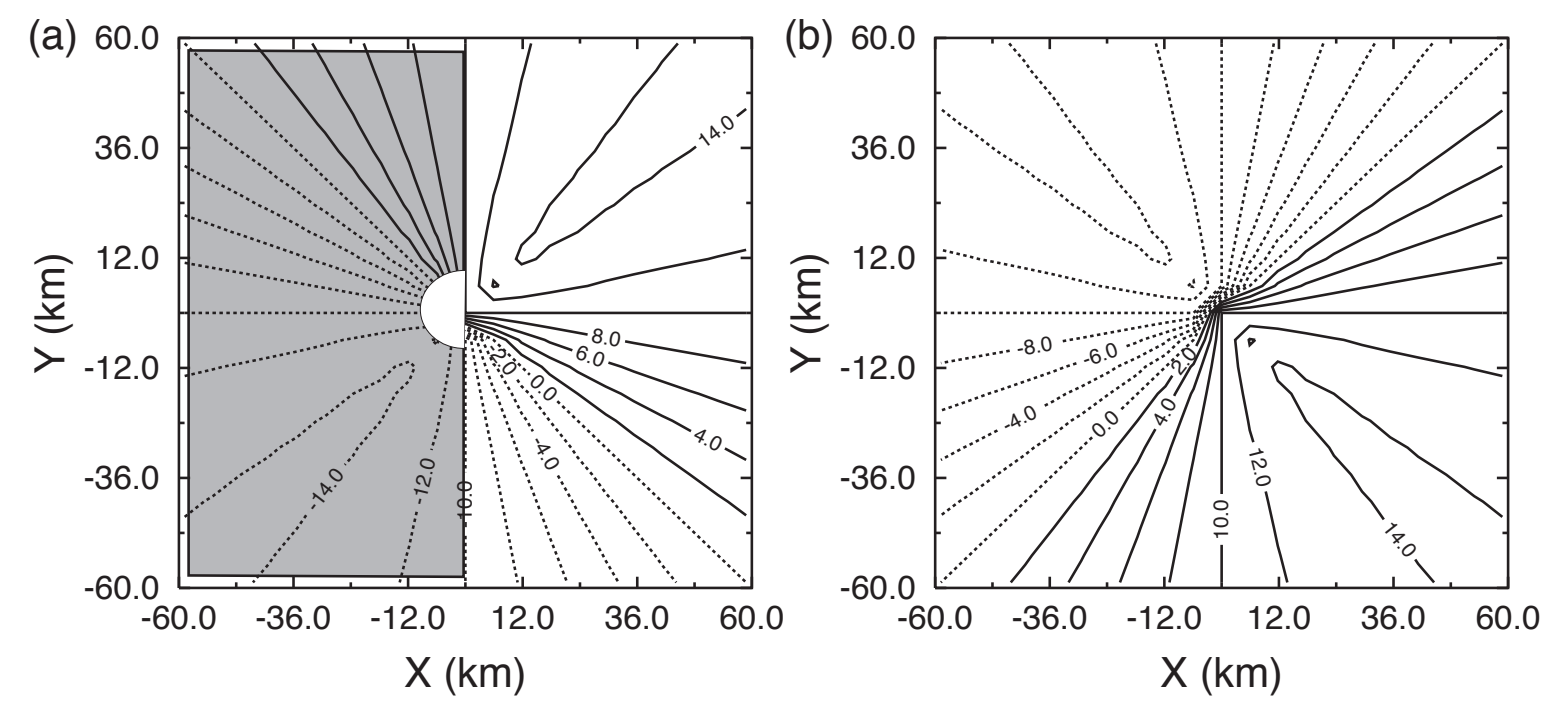

Fig. 2. Idealized true radial-wind field given by $U \cos \beta+V \sin \beta$ (a), and true tangential-wind field given by $-U \cos \beta+V \sin \beta$ (b). Contours are plotted every $2 \mathrm{~m} \mathrm{~s}^{-1}$ with solid for non-negative and dotted for negative values. Shaded area in (a) shows the coverage of observations. 

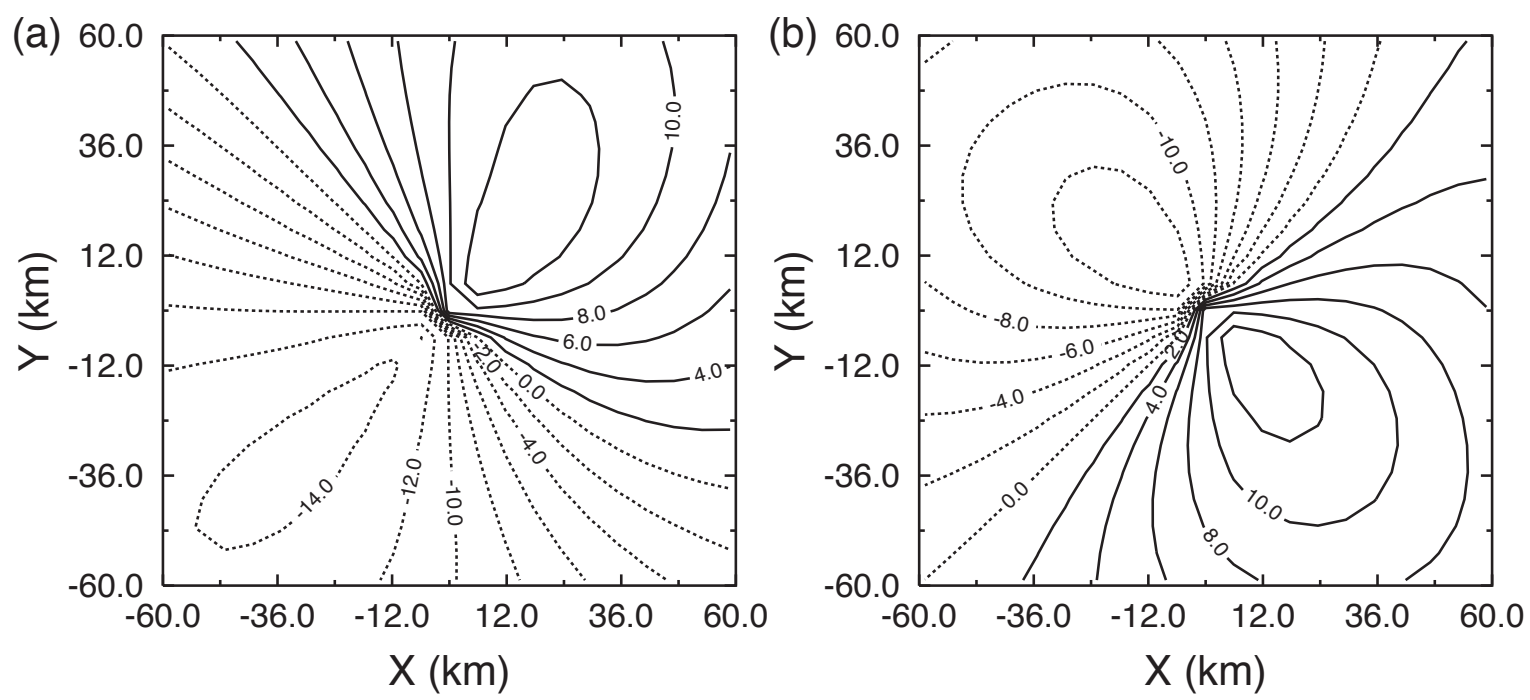

Fig. 3. As in Fig. 2 but for analyzed radial-wind field (a), and tangent-wind field (b) with $L=60 \mathrm{~km}$. 

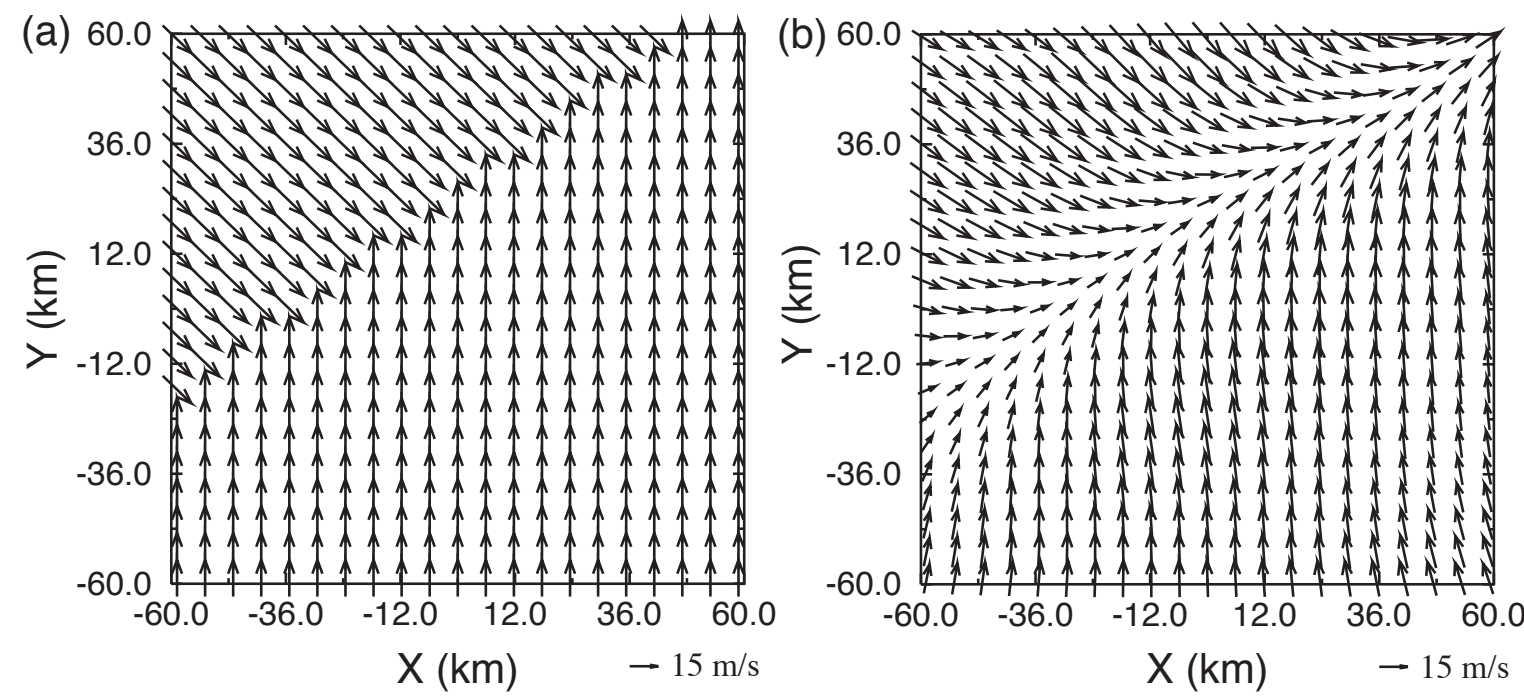

Fig. 4. True vector wind field (a) and analyzed vector wind field (b) in the idealized case of convergent flow. The true winds in (a) have constant values of $(U, V)=(0,2)$ and $(15,-15) \mathrm{m} \mathrm{s}^{-1}$ on the southeast and northwest sides, respectively, of the convergence line. 

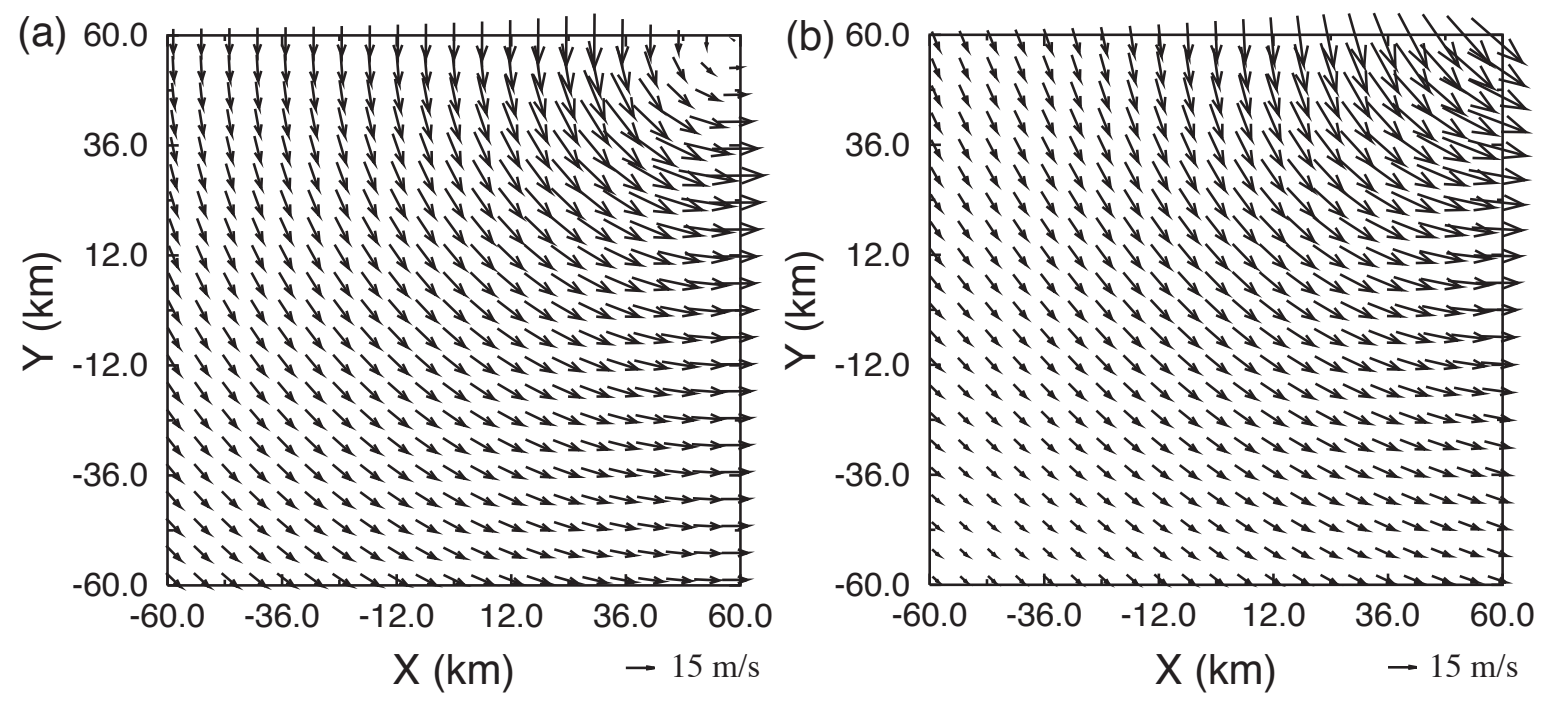

Fig. 5. True vector wind field (a) and analyzed vector wind field (b) in the idealized case of curvy flow. See text for details. 


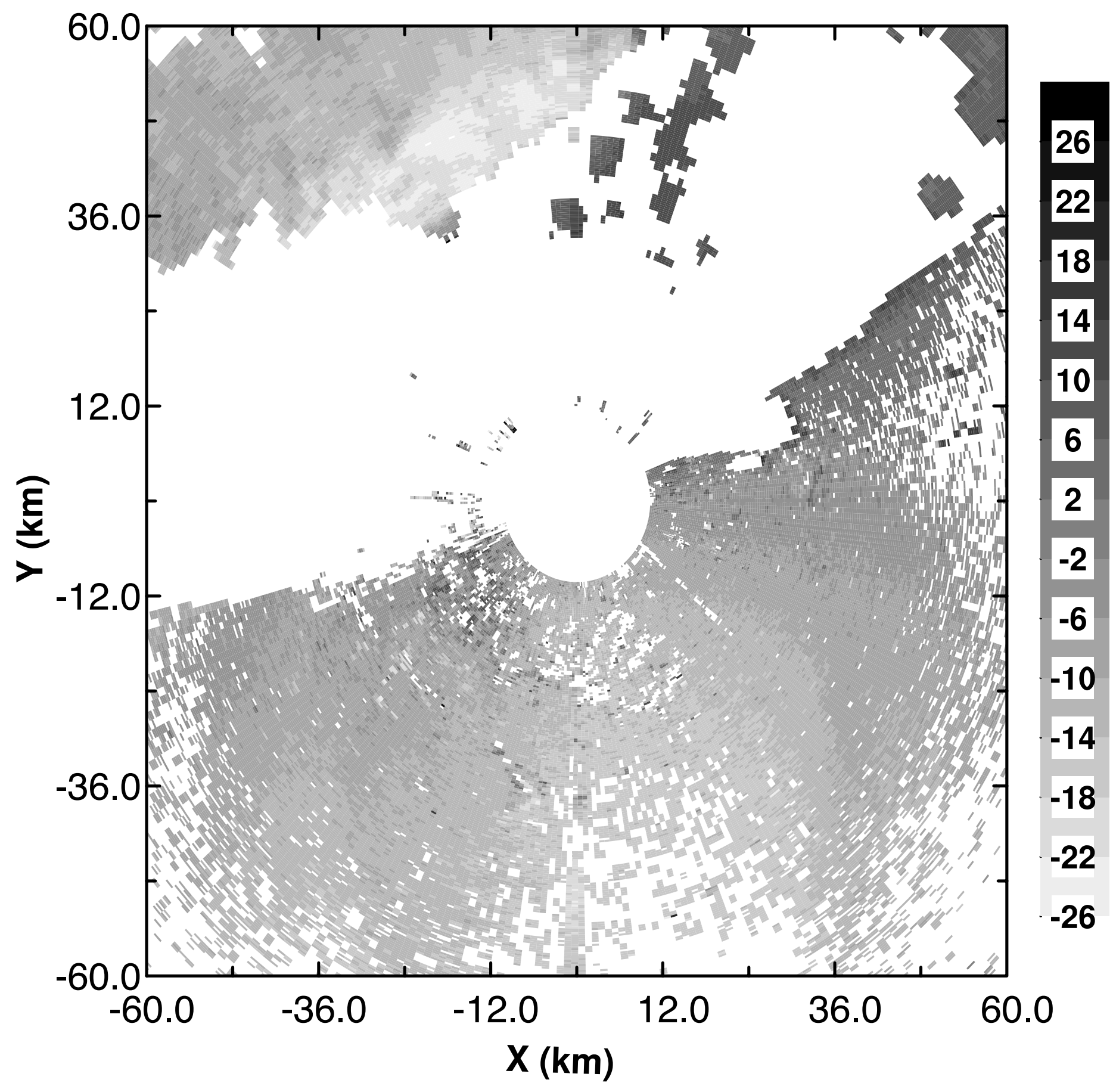

Fig. 6. Dealiased radial velocities on the conical surface of 0.5 degree elevation angle over the area of $120 \square 120 \mathrm{~km}^{2}$ centered at the radar. The raw radial velocity data were collected by the KTLX radar 0040 UTC on 16 June 2002 for a surface cold front over the area of Oklahoma state. 

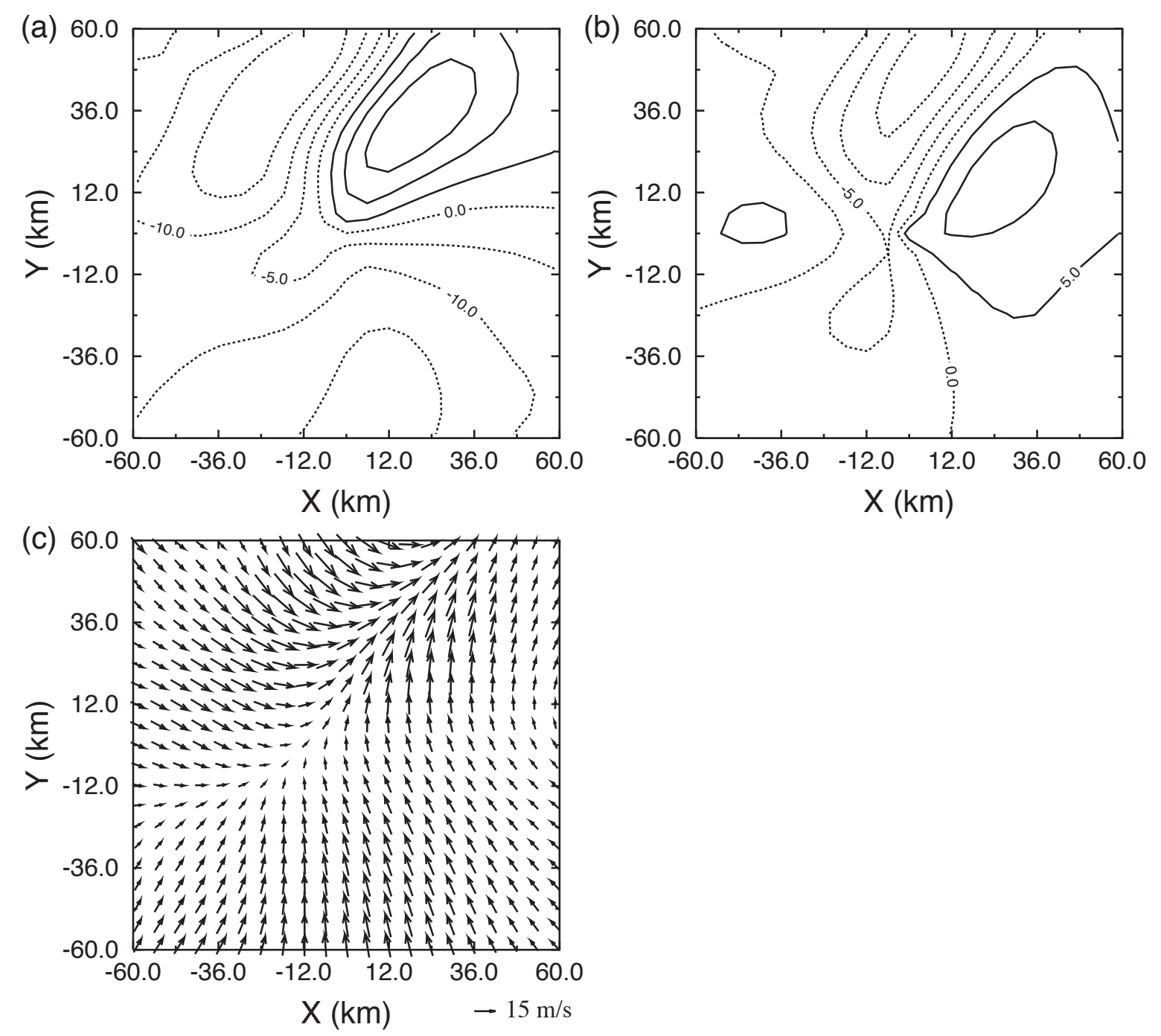

Fig. 7. Analyzed radial-wind field (a), tangent-wind field (b), and vector winds (c) for the real case. 